Mediterránea, 5 - Págs. (67 - 96), Año 1981

\title{
SOBRE NIDOS AÉREOS DE RATA NEGRA, Rattus rattus (Linnaeus, 1758), EN EL NARANJAL SAGUNTINO (VALENCIA)
}

\author{
Por F. V. FAUS y J. R. VERICAD(1)
}

\section{INTRODUCCION}

Las investigaciones sobre roedores comensales como la rata negra (Rattus rattus), la rata parda (Rattus norvegicus) y el ratón doméstico (Mus musculus) se han centrado, sobre todo, en aspectos de su control por su importancia en la salud pública y en la economía. La revisión de BROOKS (1973) sobre roedores comensales a nivel mundial, así como las referencias que contiene, constituyen un buen ejemplo de ello.

ZIMMERMANN (1953) ya señalaba la urgencia de nuevas investigaciones sobre $R$. rattus en el área mediterránea, donde al contrario que en Centroeuropa es menos comensal. Así, JIRSIK (1955) para Checoslovaquia y MEYLAN (com.verb.) para Suiza indican el estricto comensalismo de esta rata que ocupa graneros y otras construcciones humanas, habitadas o no, encontrándosela sólo muy excepcionalmente en la naturaleza. En cambio, SAINT-GIRONS (1973) y LE LOUARN y SAINT-GIRONS (1977) resaltan que en la región mediterránea de Francia es donde puede observarse su biotopo natural. NIETHAMMER y MARTENS (1975) inciden en lo mismo, asi como las publicaciones de FELTEN y STORCH (1970), TOSCHI (1965) y SANTINI $(1972,1977)$ para Italia, NIETHAMMER (1971), ONDRIAS (1965) y

(1) Centro Pirenaico de Biología experimental. Apartado 64, JACA (Huesca) 
WETTSTEIN (1941) para Grecia, FELTEN et al. (1971) para Anatolia occidental, AHARONI (1932) y ATALLAH (1978) para Palestina y, por último, BLANC et. al. (1962), BROSSET (1960), CABRERA (1932), LATASTE (1885), SAINT-GIRONS y PETTER (1965) para el Magreb.

Sobre $R$. rattus en Iberia se confirma la misma opinión con los trabajos de CABRERA (1914), LEHMANN (1969), VALVERDE (1960), VERICAD (1970) y VERICAD y MEYLAN (1973). A pesar de la recomendación de Zimmermann, ninguna de las referencias aquí expuestas, muchas de ellas posteriores a 1953, estudian con profundidad la biología de $R$. rattus. Normalmente, dado que se trata en su gran mayoría de fáunulas o faunas regionales o nacionales, se la cita en ellas de manera colateral, indicándose sólo los lugares de captura y el estado biológico de los ejemplares capturados. Algunos de estos trabajos discuten aspectos de nomenclatura de $R$. rattus en Europa y el área mediterránea, que todavía no están claros, así como aspectos biométricos.

Según lo que conocemos, solamente los escritos de $\mathrm{KAH}$ MANN y HAEDRICH (1957) y KAHMANN y ÇAGLAR (1970) se refieren exclusivamente a la rata negra en la región mediterránea. El primer trabajo presenta y discute los resultados obtenidos de las observaciones y capturas realizados de $R$. rattus en la isla de Córcega. El segundo contribuye al conocimiento de los hábitos alimentarios de la especie, resaltando las contradicciones que sobre este tema aparecen en los artículos publicados hasta aquel momento.

Si sobre la biología y requerimientos ecológicos de $R$. rattus el conocimiento que se tiene es tan escaso y fragmentario, como se deduce de la revisión que acabamos de hacer, sobre el tema que nos ocupa, cual es, el estudio de los nidos aéreos de $R$. rattus, es fácil de suponer que aún hay menos antecedentes.

La capacidad de $R$. rattus para llevar vida arborícola es conocida de antiguo. Los nidos aéreos son consecuencia de esa vida arborícola. Algunas de las referencias citadas hasta ahora hablan de ello, pero sin entrar en gran detalle. Así, LATASTE (1885) dice que en los oasis saharianos ha capturado $R$. rattus en la parte alta de las palmeras; CABRERA (1932) hace notar que en Marruecos ha encontrado esta rata en sitios con arbolado. Dice textualmente: "...la he obtenido en bosquetes de adelfas, siendo seguramente un animal arborícola como lo es en España el $R$. r. frugivorus". CABRERA (1914) señala que $R$. r. frugivorus en Iberia anida generalmente en árboles aunque se establece también en graneros y palomares. LE LOUARN y SAINT-GIRONS (1977) hacen notar que $R$. rattus en la zona mediterránea se instala en arboledas y espesuras donde construye un nido esférico con fragmentos vegetales o viven en madrigueras. NIETHAMMER (1970) describe someramente los nidos esféricos de este animal constituídos por hojas sueltas de lentisco y alcorno- 
que, y situados a metro y medio del suelo en un zarzal. Añade, además, que estos nidos, de las cercanías de Lisboa, son similares a los que $R$. rattus hace en el Mediterráneo. También, ATALLAH (1978) describe nidos aéreos de $R$. r. frugivorus sobre acacias, formados por hojas y tronquillos del mismo árbol, y localizados a más de dos metros de altura. Observó, asimismo, madrigueras del mismo animal en la base del árbol.

En fin, otros autores señalan igualmente la construcción de nidos aéreos por parte de la rata negra, cuya composición y altura varían según los materiales que tenga a disposición y del árbol soporte. KAHMANN y HAEDRICH (1957) dedican parte de su trabajo a los nidos de $R$. rattus en la isla de Córcega, de los cuales encontraron cerca de un centenar en diferentes biotopos. Son los autores más explícitos y hablan sobre el soporte de los nidos, altura, materiales de construcción utilizados y número de individuos presentes.

En nuestro trabajo, al contrario que en el de los autores precedentes, el material proviene de un único biotopo (naranjal), recogido tras búsqueda exhaustiva en el área de estudio. Ello ha permitido generalizar la tendencia en la construcción de nidos aéreos por parte de $R$. rattus en aspectos tales como dimensiones, composición cualitativa y cuantitativa, orientación, situación en la copa de los naranjos y distribución espacial de los nidos.

\section{AREA DE ESTUDIO.}

La zona estudiada, descrita ya anteriormente por GILDELGADO y ESCARRE (1977) y GIL-DELGADO et al.(1979), está situada en el término municipal de Sagunto, en la provincia de Valencia, concretamente en la partida de Montíver que corresponde a las cuadriculas UTM YJ3597 e YJ3697. La parcela ocupa una superficie de 16.919 hectáreas y está constituida por 23 huertos dedicados exclusivamente al cultivo del naranjo. Tiene una altitud de 30 metros sobre el nivel del mar y se encuentra a una distancia de 4 kilometros de la línea de costa mediterránea y a 2,5 de la ciudad de Sagunto.

La totalidad de los árboles frutales son naranjos (Citrus aurantium) de diferentes "variedades" (clementina, navel, navelina, sanguina, satsuma y valencia). Hay también unos pocos nisperos y algún limonero. La altura de los naranjos varia de 2 a 5 metros, exceptuando los plantones que son mucho más pequeños. La distancia entre ellos oscila de 4 a 5 metros, y se distribuyen homogéneamente a modo de malla cuadrangular. Puede ocurrir que falte un naranjo 0 bien que esa falta se sustituya por un plantón. El estrato herbáceo lo componen no menos de 50 especies (GIL-DELGADO et al., 1979). 
Durante el período de tiempo comprendido entre el mes de diciembre del año 1977 y diciembre de 1978, en el área de estudio ya descrita, se recogieron un total de 119 nidos aéreos de $R$. rattus. En cada estación del año se efectuaron dos búsquedas sistemáticas y exhaustivas de nidos, árbol por árbol, lo que representa un total de ocho búsquedas o barridos y 60 días completos de trabajo de campo.

Se han distinguido cuatro tipos de nidos: A) nidos aéreos de $R$. rattus, construidos con material de naranjo, de estructura completa y en uso; B) nidos aéreos de $R$. rattus, elaborados con material de naranjo, abandonados e incompletos en su estructura; $C)$ nidos de gorrión común (Passer domesticus) ocupados por $R$. rattus; y D) nidos de mirlo común (Turdus merula) utilizados también por $R$. rattus. En la TABLA I se indica el número de nidos de cada tipo recogidos por estación y en total.

Una vez localizado el nido, se le numeraba, señalando con un subíndice la clase a la que pertenecía, tomándose también su situación en el campo por medio de unas coordenadas preestablecidas que correspondían a cada árbol, y datándose la fecha de recogida.

Ya situado el nido espacial y temporalmente se anotaban los siguientes datos:

a) Dimensiones del naranjo soporte del nido: At (altura total del naranjo, desde el suelo a la rama más alta), Rn (radio de la copa del naranjo, tomado desde el tronco a la rama más exterior en el sentido del emplazamiento del nido), $\mathrm{Cb}$ (cobertura, definida por el cociente entre el diámetro mayor y menor de la copa del naranjo) y Dt (diámetro del tronco del naranjo, calculado a partir del perímetro del mismo el cual se tomaba por la base del tronco a una distancia de 20 centímetros del suelo).

$\begin{array}{lccccc} & \text { INVIERNO } & \text { PRIMAVERA } & \text { VERANO } & \text { OTOÑO } & \text { ANUAL } \\ \text { A } & 22 & 20 & 6 & 12 & 60 \\ \text { B } & 5 & 16 & 12 & 12 & 45 \\ \text { C } & - & - & 1 & 5 & 6 \\ \text { D } & 2 & 3 & - & 3 & 8 \\ \text { Total } & 29 & 39 & 19 & 32 & 119\end{array}$

TABLA I. - Número de nidos aéreos de $R$. rattus recogidos estacional y anualmente, según cada tipo considerado. 
b) Dimensiones de la situación espacial del nido respecto al árbol soporte: Rne (radio nido-eje del tronco, cuya distancia en línea recta va desde el centro imaginario del tronco al centro del nido), Ans (altura o distancia que va desde el centro del nido al suelo, perpendicularmente) y Or (orientación del nido, tomada desde la base del naranjo y en referencia al Norte).

c) Dimensiones propias del nido: Dm (diámetro mayor), Dm' (diámetro menor, perpendicularmente al primero), $\mathrm{H}$ (alzada o altura del nido), Sb (número de ramas sobre las que se emplaza el nido), $V$ (volumen hipotético calculado mediante el producto $\mathrm{Dm} \times \mathrm{Dm}$ ' $\times \mathrm{H}$ ) y Pf (peso en fresco del nido).

Las dimensiones se dan en centímetros, la orientación en grados y radianes, el peso en gramos y el volumen en centímetros cúbicos.

Por otro lado, también se observaba la posible presencia de madrigueras, localizándose al pie del naranjo analizado en la mayoría de los casos y/o en los árboles cercanos, tomándose los siguientes datos: $\mathrm{dm}$ (diámetro mayor de la boca de entrada de la madriguera), dm' (diámetro menor de la boca de entrada de la madriguera) y $f$ (profundidad de la entrada de la madriguera).

Los materiales de construcción del nido fueron analizados cualitativa y cuantitativamente. Se distinguieron: ramilletes $(R)$, tomándose la longitud total de la rama principal (l.t.), la anchura por la base de rotura (ampl.) y el número de hojas (n. ${ }^{\circ}$ h.): y, ramitas (r), sin hojas, sobre las que se media la longitud total (l.t.), el espesor por el extremo de rotura (ampl.) y el número de bifurcaciones $\left(n .^{\circ}\right.$ b.).

Para el estudio de la distribución espacial de los nidos se levantaron mapas de trabajo a partir de un plano a escala 1: 2.000 , facilitado por la Hermandad de Labradores de Sagunto. Se hizo uno para cada huerto, a escala mitad del original, en los que se representaban los frutales.

\section{DIMENSIONES DEL NIDO.}

Se han estudiado las seis dimensiones del nido aéreo de $R$. rattus, ya descritas con anterioridad en el apartado de material y métodos, y que son: Dm, Dm', $\mathrm{H}, \mathrm{Sb}, \mathrm{V}$ y $\mathrm{Pf}$.

En la TABLA II se dan los valores mínimo $(\mathrm{mn})$ y máximo $(\mathrm{mx})$, la media artimética $(\bar{X})$ con su error estándar (sm) y la desviación típica (s) de las dimensiones de los 60 nidos del tipo A considerados. Solamente en una dimensión, Dm, se ha encontrado que el valor calculado de $\mathrm{X}^{2}$ corresponde a $\mathrm{p}>0.05$, y la distribución de los datos se ajusta bien a una distribución normal, lo cual no ocurre con las de- 


\begin{tabular}{lrrrrrc} 
& Dm & \multicolumn{1}{c}{ Dm' } & \multicolumn{1}{c}{ H } & Sb & V & Pf \\
$\mathrm{mn}-\mathrm{mx}$ & $10-50$ & $8-40$ & $7-51$ & $1-10$ & $560-59400$ & $14.31-1090.07$ \\
$\overline{\mathrm{x}}$ & 26.48 & 19.43 & 18.73 & 5.00 & 11739.08 & 190.44 \\
$\mathrm{sm}$ & 1.08 & 0.91 & 1.12 & 0.27 & 1608.07 & 28.85 \\
$\mathrm{~s}$ & 8.33 & 6.97 & 8.63 & 2.05 & 12351.84 & 221.63
\end{tabular}

TABLA II. - Valores mínimo $(\mathrm{mn})$ y máximo $(\mathrm{mx})$, media aritmética (X) con su error estándar (sm) y desviación típica (s) de los 60 datos de los nidos aéreos tipo A de $R$. rattus para cada una de las seis dimensiones analizadas.

más dimensiones. En cuanto al número de ramitas que constituyen el soporte del nido, $\mathrm{Sb}$, en cuyo análisis los grupos de números se han asimilado a clases de frecuencia, podemos aceptar igualmente que se ajusta bien su distribución a una normal. Sin embargo, para las distribuciones de los datos de $\mathrm{H}$ y $\mathrm{Dm}$ ' debemos rechazar la normalidad, con lo cual podemos suponer que vienen determinadas por unas pautas de comportamiento en la construcción del nido, existiendo cierta selección en estas dimensiones. $V$ y $P f$ son variables que dependen de las anteriores y, por tanto, tampoco se distribuyen normalmente. No obstante, se ha de tener en cuenta que bajo la denominación de nidos $A$ se hallan agrupados nidos aéreos comunitarios, de reproducción e individuales, por lo que las conclusiones aquí expresadas deben considerarse como provisionales.

$\begin{array}{lccccc} & \text { INVIERNO } & \text { PRIMAVERA } & \text { VERANO } & \text { OTOÑO } & \text { ANUAL } \\ \text { Dm } & 41.7 & 29.1 & 23.5 & 19.5 & 31.5 \\ \text { Dm' } & 40.2 & 40.3 & 24.0 & 24.8 & 35.9 \\ \text { H } & 55.2 & 32.6 & 27.8 & 41.5 & 46.1 \\ \text { Sb } & 35.7 & 51.5 & 31.0 & 36.1 & 41.0 \\ \text { V } & 119.5 & 105.1 & 62.4 & 94.3 & 105.2 \\ \text { Pf } & 108.5 & 100.5 & 50.0 & 94.7 & 116.4\end{array}$

TABLA III.- Valores estacionales y anuales del coeficiente de variación para las seis dimensiones analizadas de los nidos aéreos tipo A de $R$. rattus, a partir de las variables originales. 
En la TABLA III se muestran los valores estacionales y anuales del coeficiente de variación para las seis dimensiones estudiadas de los nidos aéreos tipo A de $R$. rattus. Generalmente, se observa un descenso progresivo en el valor del coeficiente de Invierno a Verano, aumentando luego en Otoño. Pudiera suceder que ésto se relacionase con la aparición de nidos con camada en los meses de reproducción y con la posterior actividad nidificadora de los ejemplares jóvenes de $R$. rattus.

\section{COMPOSICION CUALITATIVA Y GUANTITATIVA DEL NIDO.}

Los nidos aéreos de $R$. rattus, de forma generalmente esférica, están compuestos en su totalidad por materiales procedentes de los naranjos como son ramilletes con hojas, ramitas y hojas sueltas, pertenecientes seguramente al mismo árbol soporte. Esporádicamente, y en cantidades inapreciables, pueden encontrarse restos animales y/o vegetales de alimentación u otros materiales de cons. trucción.

Se ha calculado el tanto por ciento respecto al peso total del nido de los elementos hallados en 16 nidos tipo A, de los que 3 se recogieron en Primavera, 2 en Verano y 11 en Otoño. En la TABLA IV damos el promedio del tanto por ciento, habierido agrupado los nidos estacionalmente. Los materiales más abundantes son los rami-

$\begin{array}{lccc}\text { ESTACIONES } & \text { RAMILLETES } & \text { RAMITAS } & \text { HOJAS } \\ \text { Primavera } & 32.46 & 8.38 & 57.80 \\ \text { Verano } & 43.14 & 16.15 & 37.50 \\ \text { Otoño } & 52.31 & 5.83 & 40.72\end{array}$

TABLA IV. - Porcentajes medios de los materiales hallados en 16 nidos aéreos tipo A de $R$. rattus, habiéndolos agrupado por estaciones.

Iletes y las hojas sueltas, mientras que, comparativamente, las ramitas sin hojas son más escasas. En cambio, el análisis de 5 nidos $\mathrm{C}$ y $4 \mathrm{D}$ hizo ver que cuando $R$. rattus ocupa un nido de gorrión o de mirlo común el aporte de materiales provenientes de los naranjos es poco frecuente y escaso.

Para el estudio de los elementos de contrucción encontrados (ramilletes, ramitas y hojas) se han tomado las variables indicadas en material y métodos. Dichos elementos proceden de 2 nidos de Primavera, 2 de Verano y 7 de Otoño, nidos todos del tipo A. En la TABLA $V$ se dan los valores del número de datos de cada serie (n), el 


\begin{tabular}{lcccccc} 
& \multicolumn{3}{c}{ RAMILLETES } & \multicolumn{3}{c}{ RAMITAS } \\
& I.t. & ampl. & n. ${ }^{\circ}$ h. & I.t. & ampl. & n. ${ }^{\circ}$ b. \\
$\mathrm{n}$ & 145 & 145 & 244 & 145 & 145 & 145 \\
$\mathrm{mn}-\mathrm{mx}$ & $1.3-25.1$ & $0.03-0.45$ & $1-35$ & $2.0-26.6$ & $0.02-0.43$ & $1-7$ \\
$\overline{\mathrm{X}}$ & 8.69 & 0.18 & 4.62 & 9.21 & 0.17 & 1.95 \\
$\mathrm{sm}$ & 0.39 & 0.01 & 0.29 & 0.43 & 0.01 & 0.12 \\
$\mathrm{~s}$ & 4.65 & 0.07 & 4.63 & 5.19 & 0.07 & 1.43
\end{tabular}

TABLA V. - Número de datos (n), valores mínimos $(\mathrm{mn})$ y máximo $(\mathrm{mx})$, media aritmética $(\bar{X})$ y su error estándar (sm), y desviación típi$\mathrm{ca}$ (s) de las medidas de los materiales de 11 nidos aéreos tipo A de $R$. rattus, respecto al total de datos.

mínimo $(\mathrm{mn})$ y el máximo $(\mathrm{mx})$, la media aritmética $(\overline{\mathrm{X}})$ y su error estándar (sm), y la desviación típica (s) de las series. Para el análisis del $n .^{\circ} \mathrm{h}$. y $n .{ }^{\circ}$ b. los grupos de números se han asimilado a clases de frecuencia. Se ha comprobado que sólo la anchura de las ramitas (ampl.) se ajusta a una distribución normal, reflejo, seguramente, del universo de donde proviene la meustra; sin embargo, dicho ajuste no se verifica con las restantes variables, lo que nos hace suponer que existe cierta preferencia, teniendo en cuenta que algunas medidas como el número de hojas de los ramilletes $\left(n .^{\circ}\right.$ h.) y el número de bifurcaciones de la ramitas $\left(n .^{\circ}\right.$ b.) son posiblemente imagen del universo muestreado.

\section{RAMILLETES}

PRIMAVERA VERANO OTOÑO ANUAL

$\begin{array}{lllll}\text { l.t. } & - & 52.2 & 47.0 & 53.5 \\ \text { ampl. } & - & 38.3 & 35.9 & 39.5 \\ \text { n. }{ }^{\circ} \text { h. } & 89.6 & 98.2 & 92.9 & 98.3\end{array}$

RAMITAS

I.t.

ampl.

N. ${ }^{\circ}$ b.
26.0

36.4

31.4
61.6

49.5

82.5
52.6

34.6

63.3
56.3

43.3

73.2

TABLA VI. - Valores estacionales y anuales del coeficiente de variación para las dimensiones analizadas de los materiales de $11 \mathrm{ni}$ dos aéreos tipo A de $R$. rattus. 
En la TABLA VI se aprecian los valores estacionales y anuales del coeficiente de variación para cada dimensión analizada de los materiales de los 11 nidos aéreos de $R$. rattus antes considerados. Estacionalmente, los valores más altos se observan en Verano y el coeficiente no experimenta grandes cambios estacionales excepto en las medidas de las ramitas cuyas oscilaciones son considerables. El incremento del coeficiente de variación durante el Verano se podría explicar puesto que una mayor variabilidad de las dimensiones de los materiales contribuiría a dar mayor consistencia y compactibilidad a la estructura del nido, las cuales son necesarias ya que en el período de estío la actividad reproductora de $R$. rattus parece ser más elevada y los nidos tienen que ser más resistentes para poder albergar, aparte de los adultos, a la camada.

\section{ORIENTACION.}

El estudio de las respuestas de orientación de los organismos o sus señales de actividad es de gran significado ecológico, ya que es por medio de aquellas respuestas que tales organismos encuentran su más favorable microclima.

Autores como KING et al. (1964), KOLLER (1955) y THORNE (1958) han demostrado que los roedores, ante cambios de temperatura ambiental, construyen nidos de diferente tamaño.

Así pues, los roedores, generalizando, pueden expresar su termopreferendum mediante la orientación del nido y/o el tañamo del mismo, con lo que se consigue no sólo la adecuada protección de los adultos sino que se compensa la escasa homeotermia de las crías (MAXWELL y MORTON, 1975, y otros), evitándose asi la mortalidad de las mismas.

Para el estudio de la orientación de los nidos aéreos de $R$. rattus hemos utilizado los datos, tomados respecto al Norte, de $102 \mathrm{ni}$ dos A y B, cuya situación se representa en la FIGURA 1, en una sección idealizada de una copa de naranjo de 3 metros de radio, para cada estación y el conjunto de las cuatro. En la TABLA VII se da el número de datos de cada serie $(\mathrm{n})$, los valores mínimo $(\mathrm{mn})$ y máximo $(\mathrm{mx})$, los promedios $(\bar{X})$ y el error estádar (sm), y la desviación típica (s). Los datos originales de orientación de los nidos se han transformado en radianes. Según los valores medios de orientación los resultados corresponden al Sureste para el Invierno y al Sur en las demás estaciones y anualmente.

Asimismo, según explica MARGALEF (1977, p. 355) se ha calculado la dirección media y la intensidad de orientación tanto estacional como anualmente. Los resultados de orientación media coinciden, como era de esperar, con los anteriores. La intensidad de orien- 

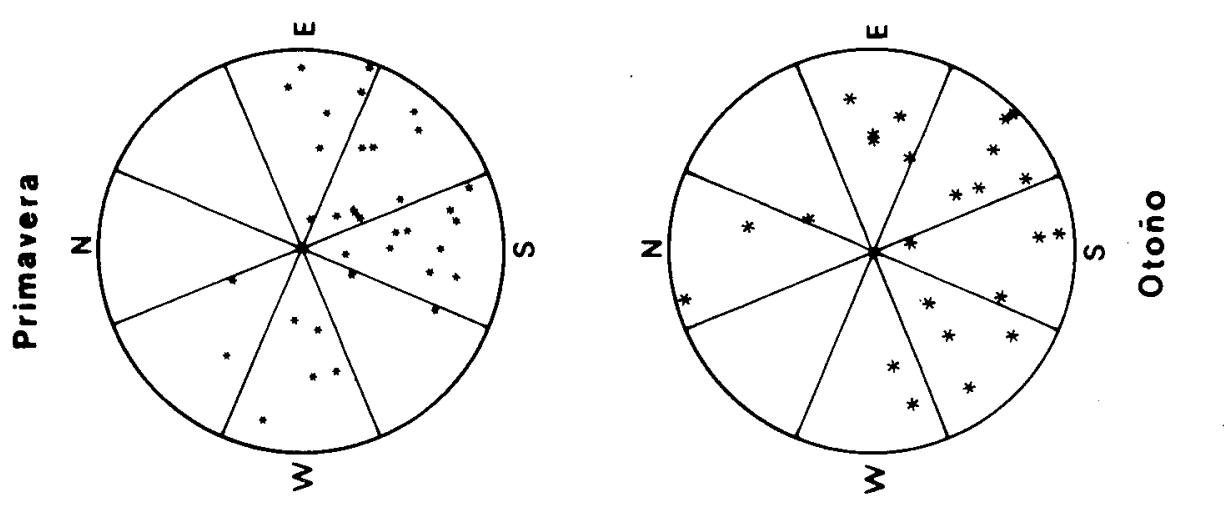

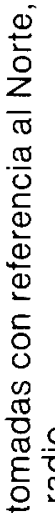

ज음

$\pm$

व

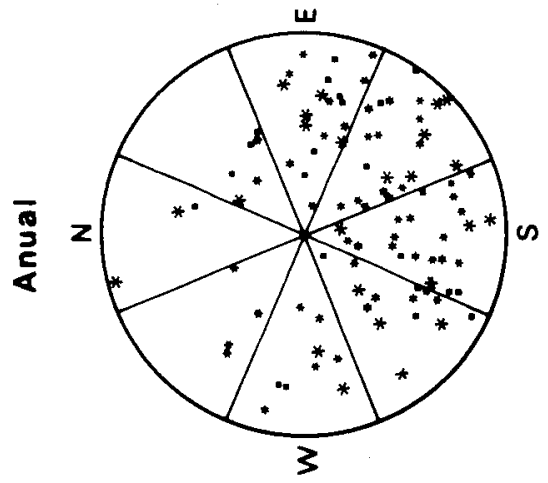

웡

$\infty \doteq$

《早

옹을 읃

으 읃

잉

용

응 엉

跤

몽

๑ 융

๘

过
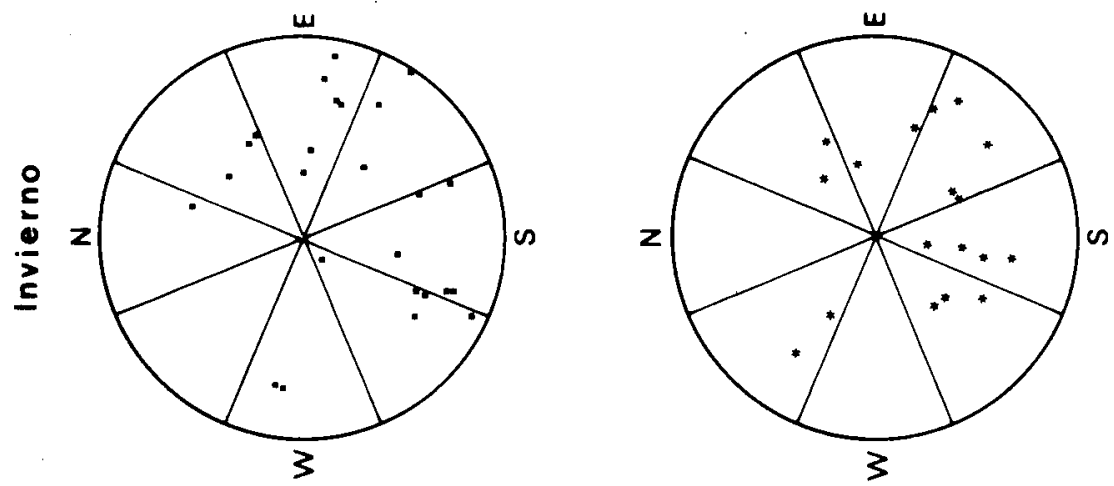

등므

동 동

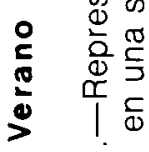

r

$\frac{\square}{\frac{\pi}{5}}$ 
INVIERNO PRIMAVERA VERANO OTOÑO ANUAL

$\begin{array}{lccccc}\mathrm{n} & 25 & 35 & 18 & 24 & 102 \\ \mathrm{mn}-\mathrm{mx} & 0.17-4.89 & 1.48-5.85 & 0.87-5.32 & 0.17-6.02 & 0.17-6.02 \\ \overline{\mathrm{X}} & 2.56 & 3.02 & 2.97 & 2.80 & 2.84 \\ \mathrm{sm} & 0.26 & 0.20 & 0.31 & 0.28 & 0.12 \\ \mathrm{~s} & 1.25 & 1.15 & 1.27 & 1.35 & 1.24\end{array}$

TABLA VII._Número de datos $(n)$, valores mínimo $(\mathrm{mn})$ y máximo $(\mathrm{mx})$, media aritmética $(\bar{X})$ con su error estándar $(\mathrm{sm})$ y desviación típica (s), para cada estación y anualmente, de las medidas de orientación de 102 nidos aéreos A y $\mathrm{B}$ de $R$. rattus, transformadas en radianes.

tación no es muy alta, siendo el valor anual de 0.46 y el más grande el de Primavera, 0.53, para una escala de 0 a 1.

La tendencia de $R$. rattus a construir los nidos en orientación básicamente Sur, tal como podríamos intuir por los resultados expuestos justamente antes, referidos a la orientación media y según la representación gráfica de los datos de orientación de la FIGURA 1 , se ha deducido significativa para el total anual de los 101 nidos $A$ y $\mathrm{B}$ considerados, ya que los valores calculados de $\mathrm{X}^{2}$ son mayores que los teóricos $(p<0.05)$. Estacionalmente, y teniendo en cuenta las orientaciones de los nidos hallados en las épocas respectivas, llegamos a la misma conclusión que en el punto anterior para Primavera y Otoño; en Invierno y Verano los valores observados de $\mathrm{X}^{2}$ son menores que los esperados $(p>0.05)$ y la tendencia en la orientación no es significativa. Pensamos que esto puede estar relacionado con la presencia de crias en los nidos, con escasa capacidad homeotérmica por tanto. En efecto, según datos propios y bibliográficos, la reproducción se presenta en Primavera, Verano y Otoño. El Invierno parece ser la época de anestro, sin crías en los nidos aéreos, por lo que, consecuentemente, la necesidad de un microcilima favorable se ve disminuída. En Verano sí que pueden encontrarse crias en los nidos, pero por la mayor benevolencia climática, esa necesidad de un microclima adecuado se ve, igualmente, disminuida.

Finalmente, hemos comprobado que los atributos "estación del añon y "orientación de los nidos" son independientes entre sí, habiendo obtenido valores de $X^{2}$ correspondientes a $p>0.05$, pudiendo concluir, en definitiva, que la tendencia de $R$. rattus a construit nidos aéreos con la misma orientación no varía estacionalmente. 
Tal como se ha indicado en los dos primeros párrafos del apartado de orientación de los nidos, tomada en referencia al eje del tronco del naranjo, aquella que se podría considerar como una expresión de las necesidades térmicas del constructor y usuario del nido. Las copas de los árboles, tanto aislados como en formaciones, gozan de un régimen microclimático propio más o menos acentuado (KALMA y STANHILL, 1972). Es Iógico pensar, pues, que la posición del nido en la copa del árbol, definida por las coordenadas del nido respecto al árbol soporte y al suelo, Rne y Ans, pueda responder a determinadas preferencias térmicas. Por ello, vamos a estudiar aquí dichas dimensiones en 101 nidos aéreos $\mathrm{A}$ y $\mathrm{B}$ de $R$. rattus, para cada estación del año y el total anual.

En la TABLA VIII se dan los valores minimo $(\mathrm{mn})$ y máximo $(\mathrm{mx})$, la media aritmética $(\bar{X})$ y su error estándar (sm), y la desviación típica (s) de los datos anuales de Rne y Ans. Se ha verificado para ambas dimensiones que los datos se distribuyen normalmente, puesto que los valores calculados de $X^{2}$ corresponden a $p>0.05$.

En cuanto a la variable Rne se ha comprobado una dependencia significativa entre el número de nidos y las distancias al tronco en las que se localizan, ya que el valor hallado de $X^{2}, 18.92$, es mayor que el teórico, 5.99 ( $p<0.05)$, existiendo una preferencia en situar los nidos entre 1 y 3 metros. Igualmente, para la dimensión Ans hay una dependencia significativa entre esta variable y el número de nidos, con un valor observado de $X^{2}$ de 9.15 , mayor que el esperado, $3.84(p<0.05)$, situándose preferentemente los nidos a alturas que varían de 2 a 3 metros.

De otro lado, hemos averiguado que los caracteres «estaciones del año", $y$ "distancias al suelo en las que se localizan los nidos" son independientes entre sí; el valor calćculado de $X^{2}$ es 1.96, menor que el teórico, 7.81 ( $p>0.05$ ), por lo que se deduce que la tendencia de $R$. rattus a ubicar los nidos a determinadas alturas dentro de la copa de los naranjos no varia a lo largo del año. También se ha verificado, además, la independencia entre los atributos «orientación de los nidos" y "distancias a las que se sitúan los nidos respecto al suelo»; los valores calculados de $X^{2}$ han resultado ser menores que los teoricos $(p>0.05)$.

Considerando las variables ahora analizadas, Rne y Ans, y las dimensiones del árbol At (altura del naranjo) y $R n$ (radio de la copa), se ha obtenido el coeficiente de correlación a partir de los datos anuales de los 60 nidos tipo $A$ de $R$. rattus. Hemos observado valores significativos del coeficiente para las relaciones siguientes: At/Ans $(r=0.32)$, At/Rne $(r=0.40)$ y $R n / R n e(r=0.63)$, mientras que pa- 


\begin{tabular}{lrr} 
& Rne & \multicolumn{1}{c}{ Ans } \\
$\mathrm{mn}-\mathrm{mx}$ & $40-300$ & $129-301$ \\
$\overline{\mathrm{X}}$ & 181.47 & 210.51 \\
$\mathrm{sm}$ & 5.99 & 3.38 \\
$\mathrm{~s}$ & 59.88 & 33.75
\end{tabular}

TABLA VIII. - Valores mínimo $(\mathrm{mn})$ y máximo $(\mathrm{mx})$, media aritmética (X) y error estándar (sm), y desviación típica (s) de los datos anuales de las dimensiones Rne y Ans de los nidos aéreos de $R$. rattus.

ra At/Rn ( $r=0.22), \operatorname{Rn} / A n s(r=0.20)$ y Ans/Rne $(r=0.04)$ no lo son. El hecho de que las dimensiones At y Ans, y Rn y Rne estén correlacionadas positiva y significativamente es evidente, puesto que son At y $\mathrm{Rn}$ las variables indicadoras del desarrollo de la copa del naranjo y, en última instancia, las que determinan los valores de Rne y Ans.

\section{DISTRIBUCION ESPACIAL DE LOS NIDOS.}

Los factores que influyen en la distribución espacial de una especie pueden agruparse en tres componentes de amplio significado: biológica (reproducción, mortalidad, migración, etc.), etológica y ambiental (heterogeneidad). Cada una de estas componentes, o su combinación, determinan la distribución espacial de cada especie en el hábitat donde vive. Lo mismo puede aplicarse, evidentemente, a determinadas señales de su actividad, como son, en este caso, los nidos.

Para el análisis del tipo de distribución de los nidos aéreos de $R$. rattus en la parcela hemos utilizado los siguientes índices:

a) Indice de dispersión de BLACKMAN (1942), $I=s^{2} / \bar{X}$, por el que aceptamos que la distribución de los elementos de una población es al azar cuando se ajusta a una distribución teórica de Poisson y el valor del cociente varianza/media es igual o cercano a la unidad, regular cuando es ostensiblemente menor y contågiosa si es mayor. Para comprobar si las desviaciones del cociente respecto a la unidad son significativas aplicamos el test de $X^{2}$ y los resultados se comparan con los valores teóricos. Este test es bueno para averiguar el tipo de distribución que siguen los componentes de la población en consideración, pero no es lo bastante eficiente para establecer el grado de contagio o agregación. No obstante, el hecho de que se verifique un buen ajuste a una distribución de Poisson no quiere decir forzosamente que los elementos se distribuyan al azar sino que no refutamos la hipótesis de que lo sea, pues puede existir una distribución contagiosa y no detectarse. 
b) Indice de MORISITA (1959), ld, a partir del cual deducimos el tamaño de los agregados existentes para cada estación del año y el total de datos del conjunto de las mismas.

c) Indice compuesto, Is/l2s, que representa el índice de Morisita calculado para una superficie dada dividido por el indice que corresponde a una superficie doble, y detecta estructuras que escapan a una formulación estadistica simple.

Para el empleo del índice de Morisita y el índice compuesto se han utilizado siete tamaños diferentes de unidad de muestreo, tratándose de cuadros de superficie creciente y doble del anterior, correspondiendo a $25,50,100,200,400,800$ y 1.600 árboles. Como el área que abarca la copa de un naranjo es aproximadamente $s=\pi 2.5$ metros cuadrados, a cada tamaño de cuadrado le corresponderá una superficie de $25 \times \pi \times 2.5^{2}, 50 \times \pi \times 2.5^{2}, \ldots, 1.600 \times \pi \times 2.5^{2}$ metros cuadrados. Se han tomado un total de 20 cuadrados para cada unidad de muestreo, escogidos al azar. Los mapas de trabajo han sido cuadriculados previamente para cada uno de los siete tamaños distintos de cuadrado.

En la TABLA IX se dan los resultados del índice de Blackman, señalando con un asterisco aquellos valores que corresponden a una distribución contagiosa, para cada tamaño de cuadrado, tanto estacional como anualmente, y a partir del número total de cuadrículas de la parcela. Según los valores calculados, en Invierno la distribución de los nidos es contagiosa para las agrupaciones de 50, 200 y 1.600 árboles, en Primavera también lo es para todos los tama-

INVIERNO PRIMAVERA VERANO OTOÑO ANUAL

$\begin{array}{llllll}25(338) & 1.15 & 2.12^{*} & 1.06 & 1.46^{\star} & 2.26^{\star} \\ 50(154) & 1.69^{\star} & 2.27^{\star} & 1.00 & 1.60^{*} & 3.20^{*} \\ 100(84) & 1.17 & 3.66^{*} & 1.16 & 2.14^{*} & 3.60^{*} \\ 200(39) & 1.84^{\star} & 3.16^{*} & 1.50^{*} & 2.28^{*} & 5.31^{*} \\ 400(21) & 1.33 & 4.70^{\star} & 1.43 & 1.48 & 4.49^{*} \\ 800(10) & 1.73 & 4.58^{*} & 1.56 & 2.11^{*} & 6.25^{\star} \\ 1600(5) & 3.17^{\star} & 2.23 & 0.38 & 2.95^{\star} & 2.35^{\star}\end{array}$

TABLA IX.-Resultados del índice de dispersión de Blackman en cada distinta agrupación de naranjos, estacional y anualmente, para los nidos aéreos de $R$. rattus. Las cifras entre paréntesis indican el total de cuadrículas en cada unidad de muestreo. Los asteriscos senalan las distribuciones que presentan contagio. 
ños de cuadrado excepto en el de 1.600, en Verano sólo se presenta contagio en la agrupación de 200 naranjos y en Otoño en todos los tamaños de cuadrado exceptuando el de 400; anualmente hay contagio en todas las agrupaciones a excepción de la de 1.600 árboles. Aquellos tamaños de cuadrado que no presentan distribución contagiosa tienen valores de I que corresponden a una distribución al azar.

En la TABLA X podemos apreciar los valores calculados para el índice de Morisita y el índice compuesto en cada uno de los tamaños de cuadrado considerados, tanto estacional como anualmente.

Deducimos que, según los resultados del índice de Morisita, en Invierno hay dos tamaños de agregado que corresponden a las agrupaciones de 50 y 400 árboles, en Primavera hay de 100 y 400, en Verano los nidos se distribuyen al azar y no hay agregación, y en Otoño el agregado preferido es el que corresponde a la agrupación de 200 naranjos. Para el total anual la agrupación óptima de los nidos se presenta en las agrupaciones de 25 y 800 árboles. Según los resultados del indice compuesto, el cual nos da una idea más aproximada

\begin{tabular}{|c|c|c|c|c|c|c|c|}
\hline $\mathrm{N} .{ }^{\circ}$ árboles & 25 & 50 & 100 & $200^{\circ}$ & 400 & 800 & 1.600 \\
\hline $\begin{array}{l}\text { Invierno } \\
\text { Id } \\
\text { Is/l2s }\end{array}$ & $\begin{array}{r}0.00 \\
-\end{array}$ & $\begin{array}{l}5.33 \\
0.00\end{array}$ & $\begin{array}{c}0.00 \\
\infty\end{array}$ & $\begin{array}{l}1.09 \\
0.00\end{array}$ & $\begin{array}{l}1.45 \\
0.75\end{array}$ & $\begin{array}{l}1.19 \\
1.22\end{array}$ & $\begin{array}{l}1.30 \\
0.91\end{array}$ \\
\hline $\begin{array}{l}\text { Primavera } \\
\text { Id } \\
\text { Is/I2s }\end{array}$ & $\begin{array}{r}0.00 \\
-\end{array}$ & $\begin{array}{l}3.33 \\
0.00\end{array}$ & $\begin{array}{l}7.86 \\
0.42\end{array}$ & $\begin{array}{l}1.80 \\
4.38\end{array}$ & $\begin{array}{l}1.81 \\
0.99\end{array}$ & $\begin{array}{l}1.56 \\
1.17\end{array}$ & $\begin{array}{l}1.25 \\
1.25\end{array}$ \\
\hline $\begin{array}{c}\text { Verano } \\
\text { Id. } \\
\text { Is/l2s }\end{array}$ & $\begin{array}{r}0.00 \\
-\end{array}$ & $\begin{array}{c}0.00 \\
\infty\end{array}$ & $\begin{array}{l}0.71 \\
0.00\end{array}$ & $\begin{array}{l}0.96 \\
0.75\end{array}$ & $\begin{array}{l}0.84 \\
1.13\end{array}$ & $\begin{array}{l}1.28 \\
0.67\end{array}$ & $\begin{array}{l}1.08 \\
1.19\end{array}$ \\
\hline $\begin{array}{l}\text { Otoño } \\
\text { Id } \\
\text { Is/l2s }\end{array}$ & $\begin{array}{r}0.00 \\
-\end{array}$ & $\begin{array}{c}0.00 \\
\infty\end{array}$ & $\begin{array}{c}0.00 \\
\infty\end{array}$ & $\begin{array}{l}1.67 \\
0.00\end{array}$ & $\begin{array}{l}1.55 \\
1.07\end{array}$ & $\begin{array}{l}1.53 \\
1.02\end{array}$ & $\begin{array}{l}1.30 \\
1.18\end{array}$ \\
\hline $\begin{array}{l}\text { Anual } \\
\text { Id } \\
\text { Is/l2s }\end{array}$ & $\begin{array}{r}8.00 \\
-\end{array}$ & $\begin{array}{l}5.27 \\
1.52\end{array}$ & $\begin{array}{l}2.52 \\
2.09\end{array}$ & $\begin{array}{l}1.51 \\
1.67\end{array}$ & $\begin{array}{l}1.37 \\
1.11\end{array}$ & $\begin{array}{l}1.54 \\
0.89\end{array}$ & $\begin{array}{l}1.24 \\
1.25\end{array}$ \\
\hline
\end{tabular}

TABLA X. - Valores calculados para el índice de Morisita (ld) y el indice compuesto (Is/l2s) en cada unidad de muestreo considerada, tanto estacional como anualmente, de los nidos aéreos de $R$. rattus. 
del tamaño de los agregados, los valores corresponden a una agrupación óptima de 200 naranjos y otro agregado más grande de 1.600 en Primavera, en Invierno el agregado preferido corresponde a la agrupación de 800 árboles, en Verano hay dos tamaños de agregado de 400 y 1.600 , al igual que en Otoño cuyos agregados preferidos son también de 400 y 1.600 naranjos. Anualmente hay dos agrupaciones optimas de 100 y 400 árboles. Como vemos, respecto a este último índice, se observan estacional y anualmente dos tamaños de agregado, predominando según los resultados estacionales uno grande de 1.600 naranjos y otro menor que oscila de 200 a 400; sin embargo, no se determina la presencia de agregados menores debido quizás al pequeño número de nidos de cada estación, sobre todo en Verano donde es bastante menor. En la FIGURA 2 podemos observar la localización exacta de los nidos aéreos de $R$. rattus en el área de estudio, así como los tamaños de agregado asignados anualmente según el indice compuesto $\mathrm{Is}_{\mathrm{s}} / \mathrm{I}_{\mathbf{2 s}}$.

No obstante, ya que cada uno de los índices aplicados para el estudio de la distribución de los nidos de $R$. rattus y la deducción del tamaño de los agregados existentes, tanto estacional como anualmente, presentan eficiencias diferentes, no podemos ser estrictos en la asignación de las agrupaciones óptimas. Considerando conjuntamente los resultados del índice de dispersión de Blackman $\left(I=s^{2} / \bar{X}\right)$, indice de Morisita (ld) y el índice compuesto ( $\left(\mathrm{l}_{\mathrm{s}} / \mathrm{I}_{2 \mathrm{~s}}\right)$ podemos deducir, a grandes rasgos, que: en Invierno hay dos tamaños de agregado de 50 y 200 árboles y otro que variaría de 800 a 1.600 como mínimo; en Primavera habría un agregado que oscilaría de 100 a 400 naranjos y otro mayor de 1.600 al menos; en Verano se observarian dos agregados preferidos que variarían de 200 a 400 árboles y otro de 1.600 como mínimo, respectivamente; $y$, en Otoño, habría dos agrupaciones preferidas, oscilando una de 200 a 400 naranjos y otra de 1.600 al menos. Anualmente se apreciaría una agregación general para todos los tamaños de cuadrado.

Por otra parte, se ha hecho una comprobación sobre el tipo de distribución que siguen los nidos áéreos de $R$. rattus dentro de los agregados. Para ello hemos considerado la agrupación de 400 árboles, la cual se dividio en un total de 16 cuadrículas, analizándose los agregados situados en cinco campos de naranjos $(5,3 a, 118 \mathrm{D}, 124$ y 127), basándonos en el índice de Blackman. Según los resultados, se deduce que, para cada estación y en todos los campos, los nidos se distribuyen al azar dentro de los agregados, excepto en Primavera donde en los campos 5 y 118D los nidos muestran una distribución contagiosa al igual que en Otoño para el campo 3a. Anualmente el contagio es general en todos los campos aquí estudiados. 


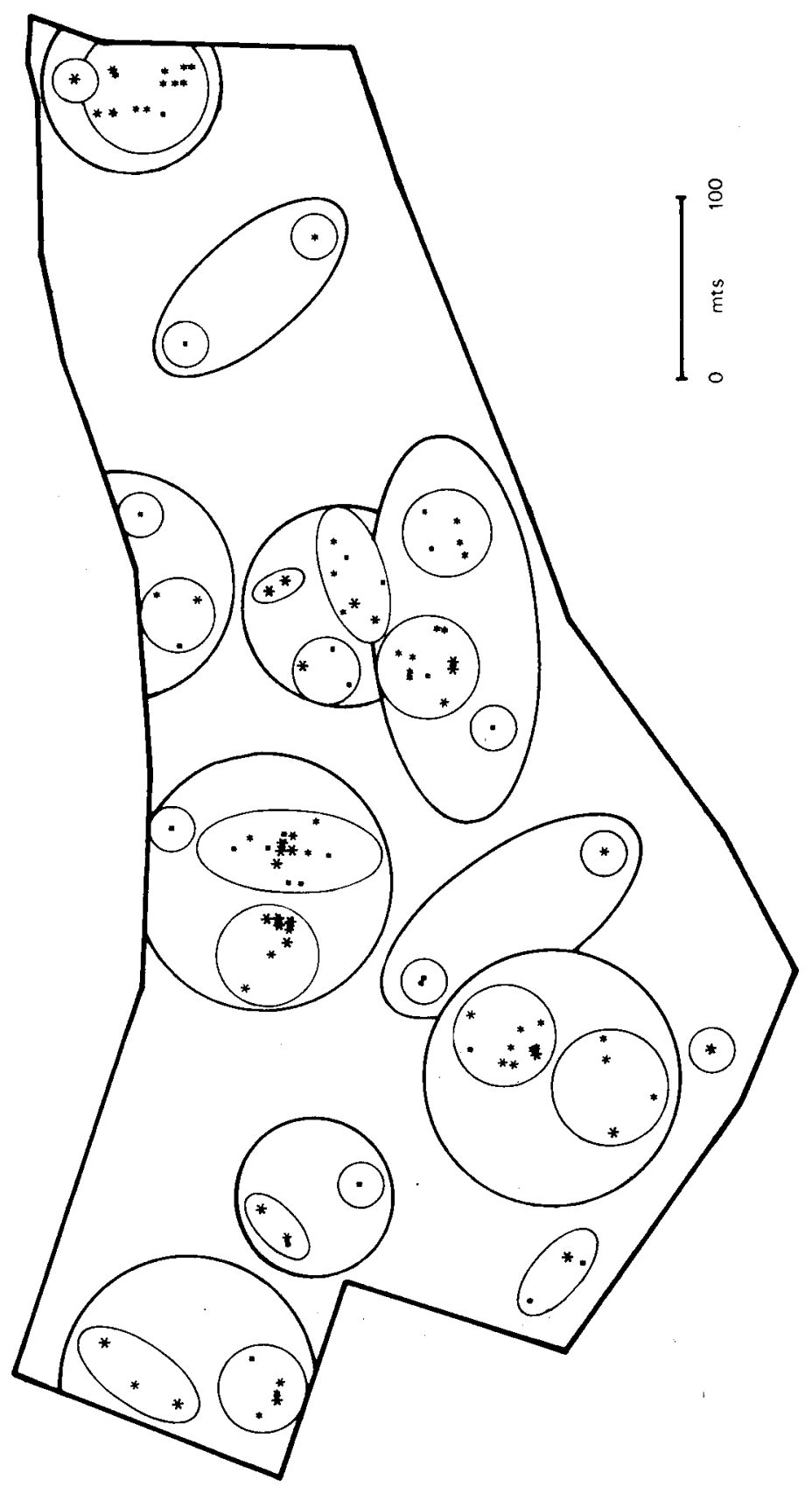

으

응

次

ते

\%

응 응

뜨응

ธิธ

宽

두응

윰

แ

这

응 운

(

응ㄹㅇㅇㅇㅇㅇ

ᄃㅇㅇㅇ

蛋高

뜸음

可

증ㅇ

宁.

등

O

응 응

원

눙

i

$\frac{\mathbb{I}}{\frac{9}{5}}$ 
Es muy probable que $R$. rattus se provea de materiales para la construcción del nido (ramilletes, hojas y ramitas) del mismo sitio donde fija su emplazamiento, porque en muchos casos se ha observado que, al quitar el nido, el lugar de nidificación aparecía desprovisto de ramas y hojas.

La técnica de construcción del nido podría consistir simplemente en la imbricación recíproca de ramilletes, hojas y ramitas hasta conseguir una estructura resistente y apropiada a las necesidades nidificadoras de $R$. rattus.

Los ramilletes y ramitas quedan cortados en sección transversal por su base de unión con la rama anterior y horizontalmente a la superficie de contacto, terminando como en cuña.

\section{MADRIGUERAS.}

Las madrigueras o guaridas de $R$. rattus, excavadas bajo tierra, constan de una boca de entrada ( $73.33 \%$ de los casos observados) o varias (de 2 a 6 en el $26.67 \%$ de las observaciones) de sección aproximadamente circular y situadas generalmente en la base del tronco del naranjo soporte del nido aéreo, en las cercanias o junto a los naranjos circundantes. Estos orificios de entrada a las madrigueras son de fácil localización por sus dimensiones considerables y por su habitual disposición cerca de los troncos de los árboles.

Su utilización debe ser complementaria con la de los nidos aéreos (BROADBOOKS, 1974), como por ejemplo en casos de inundación cuando el riego de los huertos, por la destrucción de uno u otro en períodos de limpieza, laboreo, poda o sulfatación de los campos, como refugio alternativo en defensa de los predadores, etc.

$\begin{array}{lccc} & d m & d m^{\prime} & f \\ \mathrm{mn}-\mathrm{mx} & 6-23 & 3-15 & 11-47 \\ \bar{X} & 10.67 & 7.22 & 20.44 \\ \mathrm{sm} & 0.70 & 0.44 & 1.63 \\ \mathrm{~s} & 3.57 & 2.26 & 8.29\end{array}$

TABLA XI._ Valores mínimo $(\mathrm{mn})$ y máximo $(\mathrm{mx})$, media aritmética (X) con su error estándar (sm), y desviación típica (s) de las dimensiones $\mathrm{dm}$, dm' y $\mathrm{f}$ de las 27 bocas de entrada halladas para las madrigueras de $R$. rattus. 
En la TABLA XI se dan, para un total de 27 madrigueras encontradas, los valores mínimo $(\mathrm{mn})$ y máximo $(\mathrm{mx})$ de las medidas, la media artimética $(\bar{X})$ con su error estándar (sm) y la desviación típica (s) de las dimensiones $\mathrm{dm}$, dm' y $f$ de los orificios de entrada a las madrigueras.

\section{OBSERVACIONES DE $R$. rattus.}

En los nidos aéreos de $R$. rattus, tanto A como $\mathrm{C}$ y $\mathrm{D}$, se realizaron las siguientes observaciones: en Invierno fue vista en 9 ocasiones, encontrando 2 parejas y 6 individuos solitarios en sendos nidos tipo A, descubriendo también un ejemplar en un nido de mirlo común; en Primavera se observó en 2 oportunidades, una con 3 ejemplares y otra de un individuo solitario, ambas en nidos A; en Verano se realizaron 3 observaciones, todas en nidos tipo $A$, hallando 2 camadas (una de 10 crias, sin presencia de adultos, y otra de 6 con la hembra) y un nido con una hembra y 2 ratas jóvenes; $y$, en Otoño, se vió a $R$. rattus en 6 ocasiones, encontrándose una pareja, una camada con 2 crías y la hembra, y 2 individuos aislados, todos en nidos $A$, hallando también otros 2 ejemplares solitrios en sendos nidos de gorrión común. Estacionalmente se verificaron, en definitiva, 9 observaciones de $R$, rattus en Invierno, 2 en Primavera, 3 en Verano y 6 en Otoño.

En la mayoria de las ocasiones, la reacción de los ejemplares, tanto adultos como subadultos, al percatarse de nuestra presencia era huir rápidamente saltando con habilidad entre las ramas al suelo o bien a otros naranjos, e incluso saltando directamente desde el nido a tierra salvando distancias de cuatro y cinco metros (ésto fue observado en tres ocasiones).

En la TABLA XII se dan las referencias de las observaciones de $R$. rattus, citando la fecha y el campo donde se llevaron a cabo, así como la "variedad" de naranja, las dimensiones At y Ans, el tipo de nido, y el sexo y la categoría de edad de los ejemplares vistos.

\section{OCUPACION DE OTROS NIDOS POR R. rattus.}

Del total de 119 nidos aéreos descubiertos de $R$. rattus, 14 eran de ave, correspondiendo 6 a nidos de gorrión común, $P$. domesticus, y 8 a nidos de mirlo común, $T$. merula. En estos últimos se han encontrado más restos de alimentación que en los nidos de gorrión, lo que nos induce a pensar que los primeros son más propicios para ser utilizados como comedero mientras que los segundos se usarían como refugio y lugar de descanso.

El aporte de materiales de construcción como ramilletes, hojas y ramitas, por parte de $R$. rattus, a estos nidos suele ser más bien escaso y poco frecuente. Tanto en los nidos de gorrión común como en los de mirlo, ocupados por rata negra, hemos hallado solamente individuos solitarios. 


\begin{tabular}{|c|c|c|c|c|c|c|}
\hline \multirow[b]{2}{*}{ Fecha y campo } & \multirow{2}{*}{$\begin{array}{l}\text { "Variedad" } \\
\text { de árbol }\end{array}$} & \multirow[b]{2}{*}{ At } & \multirow[b]{2}{*}{ Ans } & \multicolumn{2}{|c|}{ Tipo de nido } & \multirow{2}{*}{$\begin{array}{l}\text { Categorí } \\
\text { de edad }\end{array}$} \\
\hline & & & & A C & $\mathrm{D}$ & \\
\hline $8 \times 11-118 D$ & valencia & 3.88 & 2.57 & $2 n n$ & & ad. \\
\hline $8 \times \| 111$ & navel & 2.84 & 1.95 & $1 n$ & & ad. \\
\hline $8 \times 11-11$ & navel & 3.25 & 2.00 & $1 n$ & & ad. \\
\hline $8 \times 11-11$ & navel & 3.47 & 2.34 & $2 n$ & & ad. \\
\hline $28 \times I I-4$ & navel & 3.50 & 2.44 & $1 n$ & & sad. \\
\hline $28 \times 11-4$ & navel & 2.55 & 2.51 & $1 n$ & & ad. \\
\hline $30 \times 11-7$ & navel & 2.87 & 2.00 & $1 n$ & & ad. \\
\hline $30 \times I I-3$ & clementina & 3.05 & 2.10 & $1 n$ & & ad. \\
\hline $24 \quad 1-6$ & navel & 3.17 & 2.11 & & $1 n$ & sad. \\
\hline 4 IV-118D & valencia & 3.83 & 2.45 & $2 n$ & & ad. \\
\hline 19 IV-11 & navel & 3.53 & 2.73 & $3 n$ & & $\begin{array}{l}2 \text { ad. } \\
1 \mathrm{sad} .\end{array}$ \\
\hline 10 VIII-5 & navel & 3.62 & 2.47 & $10 n$ & & $\mathrm{cr}$. \\
\hline 22 VIII-118D & valencia & 3.57 & 2.67 & $1 q, 6 n$ & & $\begin{array}{l}\text { qad. } \\
\text { 6juv. }\end{array}$ \\
\hline $7 \mathrm{IX}-3$ & clementina & 3.31 & 2.61 & $1 @, 2 n$ & & $\begin{array}{l}\text { o ad. } \\
\text { 2juv. }\end{array}$ \\
\hline $4 \times-124$ & navel & 3.68 & 2.00 & $10^{\prime}, 1 \mathrm{n}$ & & ad. \\
\hline $4 \times-124$ & navel & 3.40 & 2.24 & $1 \%$ & & ad. \\
\hline $4 X-124$ & navel & 3.50 & 1.83 & $1 n$. & & juv. \\
\hline $11 X-11$ & navel & 3.47 & 1.90 & $\begin{array}{c}1 q, 2 \mathrm{n} \\
.\end{array}$ & & $\begin{array}{l}\text { Q ad. } \\
2 \mathrm{cr} .\end{array}$ \\
\hline $9 \times \mathrm{XI}-125$ & clementina & 3.50 & 2.52 & $1 n$ & & ad. \\
\hline $9 \times 1-125$ & clementina & 3.53 & 2.95 & $1 n$ & & ad. \\
\hline
\end{tabular}

TABLA XII.-O Observaciones de $R$. rattus en los nidos aéreos tipo A, $C$ y D. Se indica la fecha y el campo de la obsevación, la "variedad" de naranjos, las dimensiones At y Ans (en metros), el tipo de nido y la categoria de edad del ejemplar observado (cr.: cria, juv.: juvenil, sad.: subadulto y ad.: adulto). También damos el sexo del individuo ( $n=$ sexo desconocido).

Por otro lado, en ningún caso se ha observado que los nidos aéreos de $R$. rattus, después de abandonados, hayan sido aprovechados por otros vertebrados.

DISCUSION GENERAL Y CONCLUSIONES.

Los nidos aéreos de $R$. rattus de la parcela de naranjal estudiada son generalmente de forma esférica, y están compuestos en su totalidad por materiales procedentes de los naranjos y, muy proba- 
blemente, del mismo árbol soporte. Los ramilletes con hojas y las hojas sueltas representan la fracción más importante en la composición del nido; las ramitas sin hojas son, comparativamente, menos abundantes. En ninguno de los nidos tipo A revisados se ha encontrado entrada alguna bien definida. Los pocos datos existentes sobre composición de nidos de KAHMANN y HAEDRICH (1957), NIETHAMMER (1970) y ATALLAH (1978), señalan que $R$. rattus utiliza diversos materiales vegetales (hojas, talljtos, ramitas) que pueden provenir del soporte mismo o no. Los nidos responden en el empleo de los materiales a los que tienen a su mayor disposición, evidentemente. Eso explicaría la uniformidad en la composición cualitativa de los nidos estudiados, pues proceden todos de un mismo biotopo.

Respecto al somero análtsis que se ha efectuado de algunas variables contínuas o discontínuas de los materiales de los nidos se ha comprobado que varias de las distribuciones de frecuencia se acomodan a una distribución normal y otras no. Las que lo hacen se debe seguramente a que provienen de un universo que presenta tal tipo de distribución, como sería el caso del grosor de las ramitas. Las variables que pueden tener más significado biológico son la longitud de los ramilletes y de las ramitas, puesto que dependen de la rata; estas variables no se acomodan a una distribución normal, lo que indicaria que por parte de la rata hay cierta selección al cortar las ramas y los ramilletes. Otras variables como el número de hojas de los ramilletes o el número de bifurcaciones de las ramitas tampoco siguen una distribución normal, siendo muy posible que aquí la rata no efectúe selección alguna y representen tan sólo el tipo de distribución del universo del que provienen. Los valores del coeficiente de variación calculados para cada estación del año, de cada una de las variables consideradas de los materiales componentes de los nidos, son más elevados en Verano que en las restantes épocas. Esto podría estar motivado por la necesidad de una mayor consistencia y compactibilidad del nido, que debe soportar por un lado el peso de los adultos y las crías, y por otro ofrecer a éstas una protección mecánica y quizá térmica.

Los nidos aéreos de $R$. rattus representan indudablemente parte de las actividades de la población productora de los mismos. Por ello, la obtención de información sobre el grado de variabilidad de las dimensiones de los nidos y su peso no deja de tener interés. Se ha visto que sólo la distribución de los datos de $\mathrm{Dm}$ y Sb corresponden a una normal; las dimensiones restantes ( $\mathrm{H}$ y $\mathrm{Dm}$ ') y las que dependen de ellas (volúmen y peso) no lo hacen. Podría deducirse aquí que $\mathrm{H}$ y Dm' vienen determinadas por unas pautas de comportamiento en la construcción del nido.

Trabajos experimentales han demostrado que las ratas, y también los ratones, construyen nidos de tamaño diferente en respuesta a cambios de temperatura ambiental (KOLLER, 1955; THORNE, 1958). El tamaño del nido puede considerarse, así, como una medida 
del comportamiento asociado a la homeotermia, el cual puede llegar a tener carácter hereditario (KING et al., 1964). Parece también claro que la actividad tiroidea, por su influencia en la actividad metabólica y la temperatura, pueda estar relacionada con la construcción de nidos de tamaño determinado (RICHTER, 1941). Además, conviene recordar aquí que las crías de homeotermos tardan un tiempo en ser capaces de regular su temperatura corporal (cf. MAXWELL y MORTON, 1975). Es claro que en nuestro trabajo los extremos citados ahora no los hemos comprobado, pero tienen un carácter sugerente que permite interpretar biológicamente algunas de las conclusiones. Así, el valor del coeficiente de variación calculado para cada una de las dimensiones básicas de los nidos saguntinos disminuye de manera general de Invierno a Verano y aumenta de nuevo en Otoño. La explicación de dicha disminución sería que a medida que se acerca el Verano la población de ratas está formada, sobre todo, por individuos prestos a reproducirse, y la construcción del nido viene determinada por las necesidades de parto y ulterior protección de las crias; o dicho de otra manera, los nidos son resultado de una pauta de conducta en la determinación del tamaño, más estricta que en otras épocas. El aumento del valor del coeficiente en Otoño indicaría que los jovenes, ya independientes, construyen nidos de acuerdo con sus necesidades, y posiblemente inexperiencia, coexistiendo en la zona de estudio con los nidos de los ejemplares adultos.

Más arriba hemos hecho unas consideraciones sobre la relación existente, comprobada por autores ya citados, entre el tamaño del nido y la temperatura ambiental o la actividad tiroidea, así como la importancia del tamaño del nido, aparte del tipo de materiales utilizados en su construcción, en la termorregulación o en la consecución de un microclima adecuado. Pues bien, el caso que nos ocupa, cual es el de los nidos aéreos de $R$. rattus, cabe pensar que estaria relacionado con los dos aspectos citados en el párrafo anterior mediante la orientación de los nidos y las coordenadas respecto al eje del tronco del naranjo y las coordenadas al suelo; las respuestas de orientación de los organismos o sus señales de actividad, como son los nidos aéreos, son de gran significado ecológico, ya que por medio de tales respuestas los organismos encientran su microclima más favorable. Por otro lado, la situación del nido en la copa del árbol soporte, definida por sus coordenadas respecto al eje del árbol y al suelo, puede ser considerada asimismo como expresión de las necesidades térmicas del constructor del nido puesto que las copas de los árboles, tanto aislados como en formaciones más o menos densas, gozan de un microclima propio más o menos acentuado (aspectos microclimáticos de la copa del naranjo han sido tratados entre otros por KALMA y STANHILL, 1972). Como consecuencia de todo ello podemos decir que tanto el tamaño del nidos como su orientación respecto al eje del árbol y sus coordenadas respecto al árbol y al suelo son los medios que de manera aislada o conjunta expresan 
las exigencias térmicas de la especie, tanto en la apropiada protección de los adultos como de las crías, cuya escasa homeotermia se ve así compensada.

Vamos a tratar ahora de interpretar adecuadamente los resultados conseguidos al estudiar la orientación y la situación espacial del nido en la copa del naranjo soporte. La orientación media de los nidos se ha encontrado que es Sur para Primavera, Verano y Otoño, así como para el conjunto de datos de las cuatro estaciones; para el Invierno la orientación media es Sureste. Las distribuciones de frecuencia de los datos de orientación siguen una distribución normal, según se ha comprobado. Hemos confirmado, por medio de estadistica de atributos, que esta tendencia a nidificar en orientación Sur es significativa para el total de datos. Asimismo, se ha comprobado que para Primavera y Otoño existe la misma tendencia significativa a nidificar en orientación Sur; en cambio, en Invierno y Verano no, aceptando la hipótesis nula de que hay independencia entre la orientación y el número de nidos. La explicación biológica más congruente en estas conclusiones se relacionaría con la presencia de crías o no en los nidos. Ya se ha visto que la época de reproducción se presenta en Primavera, Verano y Otoño. Así, en Verano el mantenimiento de un microclima adecuado para la protección de las crias no es necesario por la benignidad climática de la estación. En Invierno, época de anestro, y, por tanto, sin crías en los nidos, las necesidades de los adultos en cuanto a microclima no son muy exigentes. Esto explicaría que en dichas dos estaciones no hay una tendencia significativa a nidificar en orientación básicamente Sur. Por último, también se ha verificado que existe independencia entre los atributos "estación del año" y "orientación", lo cual significa que la tendencia a construir nidos con la misma orientación no varía estacionalmente.

Respecto a la situación espacial del nido en la copa del árbol soporte cabe comentar que los resultados a que se ha llegado son de más difícil interpretación. Ello puede ser debido, por un lado, al desconocimiento que tenemos del microclima de la copa del naranjo en las densidades que se presenta en Sagunto, y por otro porque se ha encontrado que existe una correlación significativa positiva entre las dimensiones del árbol soporte (altura y radio medio de la copa) y las coordenadas del nido respecto al eje del tronco del naranjo y al suelo.

Tanto los individuos de una población como los restos o señales de su actividad, en este caso los nidos aéreos, pueden presentarse en tres tipos básicos de distribución espacial: al azar, uniforme y contagiosa. El primer paso a dar es conocer qué tipo de distribución siguen esos individuos o señales, y el segundo sería develar qué causas determinan el tipo de distribución hallado.

Normalmente, las distribuciones uniformes o regulares son ra- 
ras en la naturaleza, presentandose casi sólo en superficies pequeñas, y son debidas, sobre todo, a comportamiento territorial. Como este no es el caso de nuestro estudio quedarían entonces dos posibilidades: azar o contagiosa. Conviene recordar aquí que la primera hipótesis que se considera es la de que los elementos de la población se distribuyan al azar, cosa que se acepta cuando los datos se ajustan a una distribución de Poisson. Sin embargo, el hecho de que haya un buen ajuste no quiere decir que los elementos se distribuyan al azar, sino que no desechamos la hipótesis de que lo sea, pues pueden distribuirse de modo contagioso aquellos elementos y no poder detectarse. Esto quiere decir que la detección de una distribución contagiosa presenta ciertos problemas, teniendo la superficie de la unidad de muestreo gran efecto sobre los resultados a los que se llegue. Además, la deducción del tamaño del agregado en una distribución contagiosa que hemos efectuado por medio de. diversos índices tampoco es fácil, pues cada indice tiene una eficiencia propia en la detección del tamaño del agregado y da sólo valores indicativos. En general, se puede decir que la distribución espacial de los nidos aéreos de $R$. rattus es contagiosa en cada una de las estaciones del año, y presenta dos niveles de agregación que varían de tamaño según las estaciones. Así, el tamaño de agregado menor sería aproximadamente de unos 5.000 metros cuadrados y el mayor de como mínimo 22.000.

De las tres componentes que influyen en la distribución espacial de los animales: biológica, etológica y ambiental, podemos decir al menos que en este caso el contagio de los nidos no está producido por una componente ambiental, como podría ser la heterogeneidad del substrato, y en este caso el substrato es ciertamente homogéneo (monocultivo). Asi pues, el origen de esta distribución contagiosa habria que achacarlo a factores biológicos o etológicos 0 quizás a ambos.

También se deduce que, por las observaciones realizadas, $R$. rattus ocupa y utiliza preferentemente los nidos de mirlo común, $T$. merula, como plataformas de alimentación o comederos, y los nidos de gorrión, $P$. domesticus, como refugio y cama ocasionales.

Ya, por último, cabría hacer algún comentario sobre el significado de los nidos aéreos de $R$. rattus y deducir sus relaciones con los nidos subterráneos excavados por los mismos animales en las cercanías del nido aéreo. El hecho de que se hayan encontrado camadas en los nidos aéreos, algunas recientemente nacidas, así como individuos solitarios $y$, a veces, adultos y/o subadultos en grupos de hasta tres individuos, sugiere que sirven como lugar de parto y cría y como refugio individual y social. Se confirman aquí los datos al respecto de KAHMANN y HAEDRICH (1957) en la isla de Córcega, ATALLAH (1978) en Palestina y SANTINI (1977) para el Parque de la Maremma en Italia. Más arriba se ha hablado de que tanto el tamaño del nido como su orientación y situación espacial podrían muy bien 
venir determinados por la necesidad de conseguir un microclima favorable, bien para las crías, bien para los adultos. GENESTVILLARD (1972) comprobó, en efecto, que la temperatura del interior de los nidos aéreos del especializado múrido africano Tamnomys rutilans se mantenia bastante constante y más alta que la temperatura exterior, la cual podía variar hasta ocho grados. El mismo autor señala que este hecho tiene gran importancia en el mantenimiento de la temperatura corporal de este roedor en sus descansos de las salidas nocturnas. El trabajo ahora citado constituye un buen punto de apoyo en algunos de los argumentos utilizados a lo largo de esta discusión.

Parece que los nidos aéreos de $R$. rattus no estarian solamente relacionados con cuestiones microclimáticas. PACKARD y GARNER (1964) consideran que, para algunos roedores como el Ochrotomys nattalli, los nidos aéreos pueden tener cierto valor adaptativo y favorecer la supervivencia frente a catástrofes como inundaciones y algunos depredadores. En el caso de los nidos aéreos del naranjal saguntino habría que buscarles valor adaptativo junto con los nidos subterráneos, los cuales suelen ser excavados por las mismas ratas en la base de los troncos de los naranjos; así, ambos tipos de nidos tendrían un uso complementario. Hay que suponer, por otro lado, que cuando se riegan los naranjales éstos quedan prácticamente inundados al igual que los nidos subterráneos; salir de ellos, al primer síntoma de inundación y trepar para refugiarse en los nidos aéreos, es la reacción lógica. Así pues, deducimos que tanto los nidos aéreos como los subterráneos presentan soluciones alternativas para aumentar la supervivencia de $R$, rattus. En el caso concreto que nos ocupa parece que los que muestran mayor valor adaptativo son los nidos subterráneos, pues de manera normal la rata negra en el área mediterránea tiende a llevar vida arborícola. Cabe preguntarse qué pasaria en las poblaciones habitantes de zonas no cultivadas y ver si presentan dicha solución alternativa, o si sólo es propia de zonas de cultivo donde existe de modo casi continuado una presión humana por parte de los labradores al echar abajo los nidos aéreos. BROADBOOKS (1974) indica que cree que las ardillas del género Eutamias son los únicos esciúridos, y, posiblemente, los únicos roedores, en los que grupos familiares utilizan a la vez nidos aéreos y subterráneos. Sin embargo, nuestro caso puede ser otro 'más. 


\section{RESUMEN.}

El estudio de diversos aspectos ecológicos sobre la nidificación aérea de rata negra, $R$. rattus, en una parcela de naranjales del municipio de Sagunto (Valencia, España) muestra una tendencia de la especie a nidificar en este tipo de cultivo.

Los nidos aéreos, de forma generalmente esférica, han presentado una composición cualitativa uniforme a base de ramilletes con hojas y hojas sueltas procedentes de los mismos naranjos; su utilización como lugar de parto y cría así como refugio individual y social es evidente. No obstante, $R$. rattus puede instalarse en nidos de gorrión, Passer domesticus, y mirlo común, Turdus merula, para usarlos como comederos y camas ocasionales, respectivamente.

La tendencia a construir nidos con la misma orientación, preferentemente al Sur, no varió estacionalmente.

Tanto el tamaño de los nidos como su orientación y situación espacial definida por las coordenadas respecto al árbol y al suelo vendrían determinados por la necesidad de conseguir un microclima favorable.

La distribución espacial de los nidos resultó ser contagiosa tanto para el total de datos como para cada época del año, presentándose dos niveles de agregación diferentes que variaron según las estaciones.

La construcción de nidos subterráneos, o madrigueras, junto a la de los nidos aéreos, se entiende como solución alternativa para aumentar la supervivencia de la especie, pues ambos son de uso complementario. 
A study of the aerial nesting of the black rat, Rattus rattus, in orange groves at Sagunto (Valencia, Spain) shows a preference of this species to nest in this kind of tree.

The aerial nests, commonly spherical in shape, have uniform qualitative composition. They are made of small leafed branches and loose leaves from the same orange tree. It is evident that they are used for the birth and rearing of the young as well as individual and social shelter. $R$. rattus also occupies house sparrow (Passer domesticus) and blackbird (Turdus merula) nests, which are used occasionally as eating and resting places.

There is a tendency to build the nest in the south part of the tree, and this does not vary seasonally.

The size of the nests and their position in the tree (nest orientation and its spatial location defined by the coordinates to the tree axis and the ground) may be chosen to provide a favourable microclimate.

The distribution of the nests within the grove appears to be contagious for the total year data, and for every season separately, at two different levels of aggregation. The size of clumps vary according to the season at both levels.

The alternative use by this species of underground nests close to the tree-nests is considered to increase the survivorship of individuals.

KEY WORDS: Aerial nests; black rat; orange grove; Sagunto, Spain. 


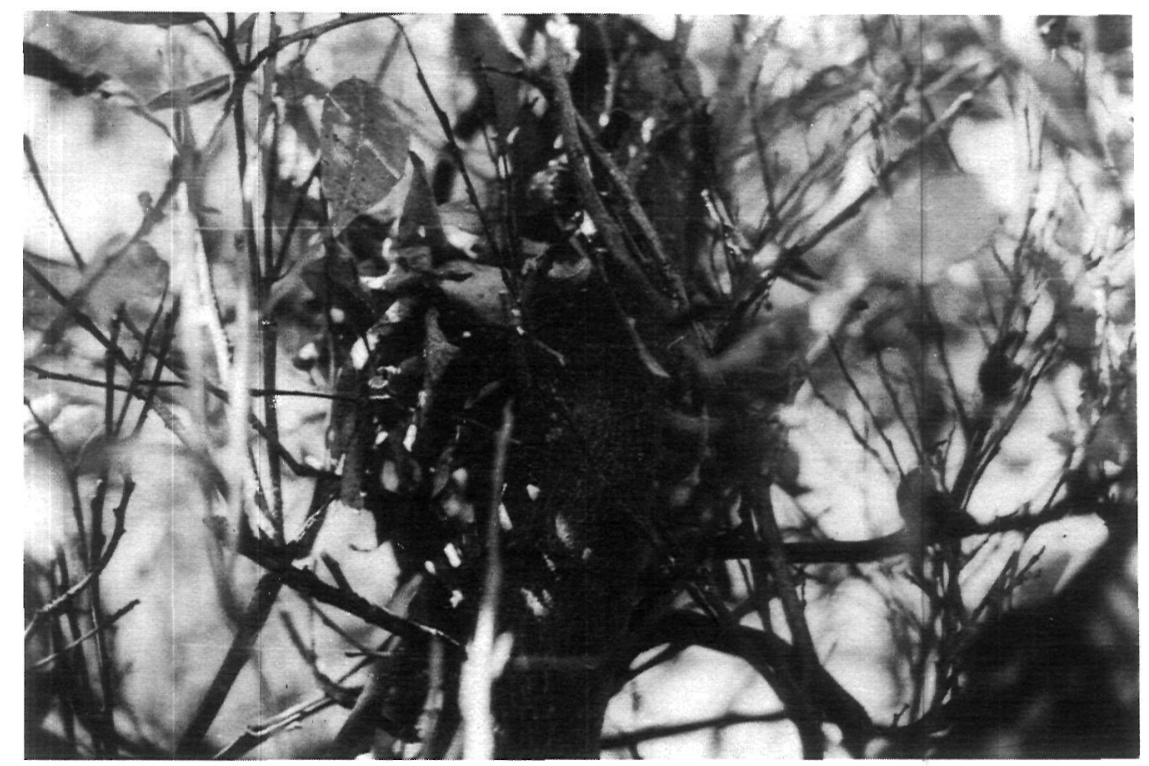

FIGURA 3.-Nido aéreo individual de $R$. rattus sobre la rama de un naranjo.

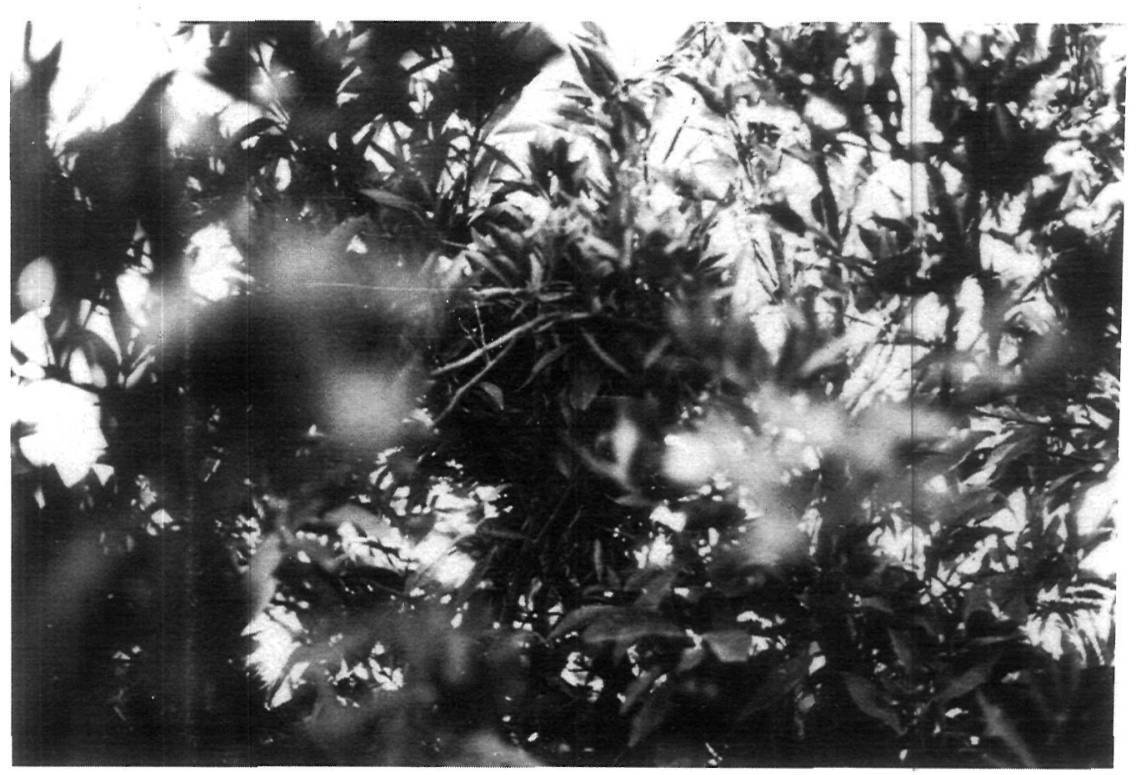

FIGURA 4.-Nido aéreo, probablemente comunitario, de $R$. rattus en el naranjal saguntino. 
AHARONI, B. (1932). Die Muriden von Palästina und Syrien. Z.f. Săugetierk. 7: 166-240. ATALLAH, S. (1978). Mammals of the Eastern Mediterranean Region: their Ecology, Sistematics ans zoogeographical Relationships. Part II. Säugetierk. Mitt. 26 (1).

BLACKMAN, G.E. (1942). Statistical and ecological studies in the distribution of species in plant comunities. I. Dispersion as a facet in the study of changes in plant population. Ann. Bot. London. N.S. 6: 351-370.

BLANC, G.; DELAGE, B.; et ASCIONE, L. (1962). Etude épidémio-écologique dans le forêt du Cherrot. Arch. Inst. Pasteur du Maroc. 6 (3): 223-292.

BROADBOOKS, H.E. (1974). Tree nests of chipmunks with comments on associated behaviour and ecology. J. of Mammalogy. 55 (3): 630-639.

BROOKS, J.E. (1973). A Review of Commensal Rodents and their control. Critical Reviews in Environmental Control. New York. 3: 405-453.

BROSSET, A. (1960). Les mammifères du Maroc Oriental. Leur répartition. Leur status actuel. Bull. Soc. Sc. Nat. et Phys. du Maroc. $40: 243-263$.

CABRERA, A. (1914). Fauna lbérica: Mamiferos. Mus. Cien. Nat. Madrid. 441 pp.

CABRERA, A. (1932). Los Mamiferos de Marruecos. Trab. Mus. Nac. Cien. Nat. Madrid. Ser. Zool. n. 57.

FELTEN, $H$. und STORCH, G. (1970). Kleinsäuger von den italienischer NittelmeerInseln Pantellarie und Lampedusa. Senckenbergiana biol. 51 (3/4): 159-173.

FELTEN, H; SPITZENBERGER, F.; und STORCH, G. (1971). Zur Klein-säugerfauna West-Anatolien. T. I. Senckenbergiana biol. 52 (6): 393-424.

GENEST-VILLARD, H. (1972). Contribution a l'écologie et l'éthologie d'un petit rongeur arboricole, Thamnomys rutilans, en République Centrafricane. Mammalia. 36 (4): 543-578.

GIL-DELGADO, J.A. y ESCARRE, A. (1977). Avifauna del naranjal valenciano. I. Datos preliminares sobre mirlo (Turdus merula L.). Mediterránea.2: 89-109.

GIL-DELGADO, J.A.; PARDO, R.; BELLOT, J.; y LUCAS, I. (1979). Avifauna del naranjal valenciano. Il. El gorrión común (Passer domesticus L.). Mediterránea. 3: 69-99.

JIRSIK, J. (1955). Die Hausratte, $R$. rattus (Linné, 1758) wieder in der Tschechoslowakei. Săugetierk. Mitt. 3: 21-29.

KAHMANN, von $\mathrm{H}$. und HAEDRICH, B. (1957). Eine untersuchung an $R$. rattus Linnaeus, 1758 (Mamm., Rod.) auf der Insel Korsika. Zoologischer Anzeiger. 158: 233-257.

KAHMANN, von H. und ÇAGLAR, M. (1970). Die Pflanzenkost der Hausratte, $R$. rattus (Linnaeus, 1758) in Mittelmeergebiet. Säugetierk Mitt. 18: 45-52.

KALMA, J.D. and STANHILL, G. (1972). The climate of an orange orchard: physical characteristics and microclimate relationships. Agricultural Meteorology. 10 (3): 185-201.

KING, J.A.; MAAS, D.; and WEISMAN, R.G. (1964). Geographic variation in nest size among species of Peromyscus. Evolution. 18: 230-234.

KOLLER, G. (1955). Hormonale und psychische Steuerung beim Nestbau weiser Mäuse. Verh. Deutsch. Zool. Ges. Freiburg. (1955): 123-132.

LATASTE, F. (1885). Etude de la faune des Vertébrés du Barbarie (Algérie, Tunisie, Maroc). Catalogue provisoir des Mammifères apélagiques sauvages. Act. Soc. Linn. Bordeaux. 34: 129-259.

LEHMANN, E. von, (1969). Zur Säugetierfauna Südandalusiens. Sitz. Ges. Naturforsch. Freunde zur Berlin. (N.F.). 9 (1/2): 15-32.

LE LOUARN, H. et SAINT-GIRONS, M.C. (1977). Les Rongeurs de France. Ann. Zool. Ecol. anim. (n. ${ }^{\circ}$ hors série). INRA. $161 \mathrm{pp}$.

MARGALEF, R. (1977). Ecología. Ed. Omega. Barcelona. 951 pp.

MAXWELL. C.S. and MORTON, M.L. (1975). Comparative thermoregulatory capabilities of neonatal ground squirrels. J. of Mammal. 56 (4): 821-828.

MEYLAN, A (1966). Liste de Mammifères de Suisse. Bull. Soc. Vaod. Sc. Nat. N. ${ }^{\circ} 321$, Vol. 69 (Lausanne, Suisse). 
MORISITA, M. (1959). Measuring the dispersion of individuals and analysis of the distributional patterns. Mem. Fac. Sci. Kyushu. Univ. Ser. E. Biol. 2: 215-235.

NIETHAMMER, J. (1970). Über Kleinsäuger aus Portugal. Bonn. Zool. Beitr. 21 (1/2): 89118.

NIETHAMMER, J. (1971). Kleinsäuger von Kithira, Griechenland. Säugertierk. Mitt. 19 (4): 363-365.

NIETHAMMER, J. und MARTENS, J. (1975). Die Gattungen Rattus und Maxomys in Afghanistan und Nepal. Z. f. Säugetierk. 40 (6): 325-355.

ONDRIAS, J.C. (1965). Die Säugetiere Griechenlanḍs. Säugetierk. Mitt. 13 (3): 109-127.

PACKARD, R.L. and GARNER, H. (1964). Arboreal nests of the golden mouse in eastern Texas. J. of Mammal. 45 (3): 369-374.

RICHTER, C.P. (1941). Behaviour and endocrine regulations of the invernal environment. Endocrinology. 2: 193-195.

SAINT-GIRONS, M.C. (1973). Les Mammifères de France et du Benelux (faune marine exceptée). Doin. Paris. $481 \mathrm{pp}$.

SAINT-GIRONS, M.C. et PETTER, F. (1965). Les Rongeurs du Maroc. Trav. Inst. Scient. Chérif. S. Zool. n. ${ }^{\circ} 31.55 \mathrm{pp}$.

SANTINI, L. (1972). Biologia e comportamento dei Mammiferi Roditori nocivi in Italia alle sostanze alimentari conservate. Ed. TEP. Piacenza. $24 \mathrm{pp}$.

SANTINI, L. (1977). Primi relievi sulla micrommalofauna terrestre (Insectivora, Rodentia) del Parco della Maremma. Informatore Botanico Italiano. 9 (2): 164-173.

THORNE, D. (1958). Shredding behaviour of the white-footed mouse, Peromyscus maniculatus osgoodi, with special reference to nest building, temperature and light. Thorne Ecol. Res. Station. Bull. No. 6.

TOSCHI, A. (1965). Fauna d'Italia. Vol. VII, Mammalia (Lagomorpha, Rodentia, Carnivora, Ungulata, Cetacea). Typ. Calderini. Bologna. $647 \mathrm{pp}$.

VALVERDE, J.A. (1960). Vertebrados de las Marismas del Guadalquivir. Arch. Inst. Acl. Almeria. 9: 9-168.

VERICAD, J.R. (1970). Estudio faunístico y biológico de los mamíferos montaraces del Pirineo. P. Cent. pir. Biol. exp. 4: 7-229.

VERICAD, J.R. et MEYLAN, A. (1973). Resultats de quelques piègeages de Micromammifères dans le Sud-est de l'Espagne. Mammalia. 37 (2): 333-341.

WETTSTEIN, O. (1941). Die Săugetierwelt der Ägäis, nebst einer Revision des Rassenkreises von Erinaceus europaeus. Ann. Nat. Hist. Mus. Wien. 52: 245-278.

ZIMMERMANN, K. (1953). Die Rodentia Kretas. Z. f. Säugetierk. 17: 21-51. 